DidÁCTICA Y CURRICULUM: REFLEXIONES EN TORNO A LA CONSTITUCIÓN DE SU NORMATIVIDAD. PÁgINAS 149-166 En REVISTA DE LA EsCuela de CIENCIAS de LA EDUCACIÓN, AÑo 11, NúMERO 10, ENERO A DICIEMBRE DE 2015. ISSN 1851-6297. ISSN EN LINEA 2362-3349.

\title{
DIDÁCTICA Y CURRICULUM: REFLEXIONES EN TORNO A LA CONSTITUCIÓN DE SU NORMATIVIDAD
}

\author{
Por Sofía Picco* \\ Universidad Nacional de La Plata, Argentina. \\ sofiaps@yahoo.com.ar
}

Recibido: 30/06/2014 Aceptado: 17/11/2014

\section{Resumen}

Nos proponemos en este trabajo postular que la "programación de la enseñanza" puede convertirse en un espacio de articulación entre la Didáctica y el Curriculum. En el marco de esta propuesta, nos interesa a su vez reflexionar acerca de las características epistemológicas que estas disciplinas poseen.

Para alcanzar nuestros propósitos, en primer lugar, conceptualizamos la Didáctica como una disciplina teórica que se ocupa de estudiar la enseñanza y orientarla a través de la elaboración de normas. Por su parte, entendemos el Curriculum como una disciplina que se encarga de estudiar el diseño, puesta en marcha y evaluación de proyectos de formación, así como también de reflexionar y resolver los problemas implicados en esas prácticas.

Reconocemos que las relaciones interdisciplinares entre la Didáctica y el Curriculum se presentan como problemáticas y con particularidades en cada país. En este sentido, en un segundo apartado del trabajo, sostenemos que la "programación de la enseñanza”, entendida como una práctica nodal del quehacer docente, puede convertirse en un espacio que reciba los aportes de ambas disciplinas para el mejoramiento efectivo de la enseñanza.

Por último, esta propuesta de articulación interdisciplinaria conlleva algunos desafíos relativos a la constitución epistemológica sobre los que es interesante reflexionar.

\footnotetext{
* Doctora en Ciencias de la Educación (Facultad de Humanidades y Ciencias de la Educación -FaHCE-, Universidad Nacional de La Plata -UNLP-). Prof. adjunta a cargo de "Didáctica y curriculum" (FaHCE, UNLP). Docente investigadora del Instituto de Investigaciones en Humanidades y Ciencias Sociales (IdIHCS, FaHCE, UNLP - CONICET). Integrante del proyecto de investigación "La formación en el ejercicio de la docencia: discursos y prácticas en el nivel primario del sistema educativo de la Provincia de Buenos Aires durante los años 2005-2012". Programa de Incentivos a la Investigación, Ministerio de Ciencia, Tecnología e Innovación Productiva de la Nación. (H659).
} 
Revista de la Escuela de Ciencias de la Educación, año 11, número 10, enero a diciembre de 2015. Páginas 149-166. ISSN 1851-6297. ISSN EN LINEA 2362-3349. DIDÁCTICA Y CURRICULUM: REFLEXIONES EN TORNO A LA CONSTITUCIÓN DE SU NORMATIVIDAD. SOFIA PICCO

\section{Palabras clave:}

Didáctica - Curriculum - Programación de la enseñanza - Relaciones interdisciplinares - Mejoramiento de la enseñanza.

\section{Abstract}

We propose in this paper postulate that "teaching programming" can become a joint space between Didactics and Curriculum. Under this proposal, we are interested in turn reflect on the epistemological characteristics that these disciplines possess.

To achieve our purposes, first conceptualized Didactics as a theoretical discipline concerned to study the teaching and to guide it through the elaboration of norms. On the other hand, we understand the Curriculum as a discipline that is required to examine the design, implementation and evaluation of training projects, as well as to reflect and solve the problems involved in those practices.

We recognize that the interdisciplinary relationship between Didactics and Curriculum are presented as problems and particularities of each country. In this sense, in a second section of the paper, we argue that "teaching programming", understood as a nodal practice teaching work, can become a space that receives contributions from both disciplines for the effective improvement of teaching.

Finally, this proposal of interdisciplinary articulation carries some challenges concerning the epistemological constitution on which is worth considering.

\section{Keywords:}

Didactics - Curriculum - Teaching programming - Interdisciplinary relationships - Improving teaching.

\section{¿Qué entendemos por Didáctica y por Curriculum?}

Comenzamos nuestro trabajo reconociendo la diversidad de sentidos que adoptan la Didáctica y el Curriculum y la disímil delimitación del campo disciplinar que cada término conlleva dependiendo del posicionamiento teóricoepistemológico que sustente el sujeto que los utiliza. Específicamente a los fines de este artículo pretendemos presentar una definición de Didáctica y otra de Curriculum que nos permita sistematizar aportes conceptuales e instrumentales para el mejoramiento de la "programación de la enseñanza" y, por ende, de las prácticas de enseñanza que llevan a cabo los docentes cotidianamente.

En primer lugar, recordamos que la Didáctica es una disciplina de larga data si consideramos que muchos autores (entre ellos: Barco, 1989; Camilloni, 1993; Martínez Bonafé, 2004) coinciden en ubicar su conformación oficial a partir de la publicación de la Didáctica Magna de Comenio en 1657 (1). Desde entonces, se ha desarrollado profusamente en países de Europa continental y de Latinoamérica. 
Revista de la Escuela de Ciencias de la Educación, año 11, número 10, enero a diciembre de 2015. Páginas 149-166. ISSN 1851-6297. ISSN EN LINEA 2362-3349. DIDÁCTICA Y CURRICULUM: REFLEXIONES EN TORNO A LA CONSTITUCIÓN DE SU NORMATIVIDAD. SOFIA PICCO

Sostenemos que la Didáctica es una teoría normativa acerca de la enseñanza. En este sentido, acordamos con Camilloni (2007) cuando plantea que se trata de una disciplina cuyo objeto de estudio es la enseñanza y que pretende describirla y explicarla así como también elaborar y fundamentar normas para colaborar con los docentes en la resolución de los problemas que las prácticas les presentan.

En este sentido y de una manera general asumimos que el conocimiento científico se ocupa de describir y explicar el objeto de estudio al que se refiere (Díaz, 1997). Sostenemos que la Didáctica describe la enseñanza cuando da cuenta de sus partes constitutivas y de sus cualidades. A su vez, decimos que se ocupa de explicar su objeto de estudio, entendiendo que se trata de explicaciones comprensivistas, en el sentido que le asignan Klimovsky e Hidalgo (1998). Este tipo de explicaciones apuntan a dar cuenta de la multiplicidad de dimensiones que intervienen en la situación a la que esperan darle inteligibilidad, además de los sentidos que los sujetos sociales como tales les asignan a sus acciones.

Por otro lado, las pretensiones normativas de la Didáctica implican la elaboración de orientaciones tendientes a la concreción de "buenas prácticas de enseñanza" en el sentido epistemológico y ético, tal como lo entiende Fenstermacher (1989).

Reconocemos la heterogeneidad de significados que porta el término "norma". No obstante, a los fines de arribar a algunos sentidos que nos permitan profundizar en la Didáctica, recuperamos a von Wright (1970) cuando dice que las normas son aquéllas que se ocupan de lo que debe y puede hacerse y de lo que no tiene que hacerse. El autor también establece una distinción útil para nuestra investigación entre norma y prescripción, siendo esta última un tipo particular de aquélla (junto con las reglas y las directrices o normas técnicas). Las prescripciones son emitidas por un sujeto que se erige como autoridad normativa, están orientadas a un sujeto normado (2) para que este último adecue a ellas su comportamiento. En este sentido, las prescripciones son órdenes o permisos dirigidos a regular la conducta de los sujetos normados.

Además de estas distinciones terminológicas, la norma y la prescripción en la Didáctica guardan una estrecha relación en nuestro país con tradiciones teóricas. La norma didáctica se define en su vinculación con los valores y con la concreción de un proyecto político-educativo; mientras que la prescripción didáctica está más asociada al tecnicismo y a la búsqueda eficiente de resultados (Davini, 1996; Camilloni, 2007).

En cuanto a la constitución de la Didáctica, cabe mencionar además que, sobreentendiendo la complejidad de la enseñanza como práctica social (Fenstermacher, 1989; Basabe y Cols, 2007; Feldman, 2010), acordamos con Camilloni (1996) cuando plantea que esta disciplina produce un corpus propio de conocimientos pero además integra y resignifica saberes provenientes de las 
Revista de la Escuela de Ciencias de la Educación, año 11, número 10, enero a diciembre de 2015. Páginas 149-166. ISSN 1851-6297. ISSN EN LINEA 2362-3349. DIDÁCTICA Y CURRICULUM: REFLEXIONES EN TORNO A LA CONSTITUCIÓN DE SU NORMATIVIDAD. SOFIA PICCO

ciencias sociales para alcanzar sus finalidades. Como lo desarrolla la autora, esta condición epistemológica le implica lidiar con diferentes problemas heredados pero como ventaja obtiene la riqueza para la explicación y la intervención que le aportan estas perspectivas teóricas.

En lo que respecta al Curriculum, sabemos que es un término polisémico. Reconocemos la existencia de diversas conceptualizaciones, algunas más tradicionales que asocian al curriculum al plan de estudios o a los contenidos que deben ser transmitidos por la escuela; otras más amplias, como pueden ser las que problematizan las relaciones entre el curriculum prescripto y el curriculum real, o aquéllas otras que miran las tensiones entre curriculum, Estado y sociedad.

Así, Díaz Barriga $(1991,1994)$ sostiene que el término curriculum emerge de la mano de la pedagogía pragmática en la sociedad industrial estadounidense y si bien aparecen algunas ideas a principios del siglo XX, el campo se desarrolla y especializa al finalizar la II Guerra Mundial. En cambio, Hamilton (1993; 1999) considera que el término curriculum aparece asociado por primera vez a las prácticas educativas en los colegios europeos protestantes del siglo XVII; mientras que Egan (2000) le asigna un sentido curricular a la educación desarrollada en los diálogos que tanto Aristóteles como Platón mantenían con sus discípulos en la antigua Grecia.

No obstante esta diversidad de definiciones, al igual que hicimos para el caso de la Didáctica, nos inclinamos por una conceptualización que considere al curriculum genéricamente como una propuesta formativa. Todo curriculum expresa aquello que una sociedad particular, en un momento histórico determinado, considera que es valioso transmitir a las jóvenes generaciones (Lundgren, 1992; Kemmis, 1993; De Alba, 1995; Salinas Fernández, 1997).

Por su parte, el Curriculum es también la disciplina que se encarga de estudiar el diseño, puesta en marcha y evaluación de proyectos de formación, así como también de reflexionar y de resolver los problemas implicados en dichas prácticas.

Por otro lado, hasta donde hemos podido indagar, no se presenta en la producción teórica curricular una discriminación entre "normativo" y "prescriptivo" (3) sino que ambos términos se utilizan indistintamente. No obstante, las reflexiones sobre las diferencias que se pueden asignar a las normas y a las prescripciones que se generan en la Didáctica, pueden ser utilizadas para el caso del Curriculum.

En este sentido, consideramos que la normatividad es inherente al curriculum y a su elaboración como propuesta formativa en tanto todo curriculum se formula para ser llevado a la práctica y, por tanto, debe orientarla hacia la consecución de las intencionalidades formativas que se propone.

Por otra parte, en lo que respecta a la teoría curricular, Stenhouse plantea que el Curriculum debe ser entendido como una ciencia normativa. "[...] Las 
Revista de la Escuela de Ciencias de la Educación, año 11, número 10, enero a diciembre de 2015. Páginas 149-166. ISSN 1851-6297. ISSN EN LINEA 2362-3349. DIDÁCTICA Y CURRICULUM: REFLEXIONES EN TORNO A LA CONSTITUCIÓN DE SU NORMATIVIDAD. SOFIA PICCO

teorías a gran escala son muy útiles como andamiaje para el avance del conocimiento, pero cuanto más satisfactorias las encontremos desde el punto de vista lógico, tanto menos probable es que sean adecuadas [...]" (1991, p.110) a las circunstancias de la práctica.

Para este autor, el curriculum se conceptualiza en la tensión compleja que se entabla entre nuestras aspiraciones y los intentos por hacerlas realidad. Consideramos que este "hallazgo" de Stenhouse y posiblemente de otros autores comprendidos en la perspectiva práctica, impide pensar al curriculum como un objeto y como una teoría que pueda abstraerse de su compromiso para con las prácticas educativas.

Cabe mencionar también el trabajo de Dussel (2010), quien avanza en el análisis del curriculum como norma. En primer lugar, para la autora el curriculum es una norma en tanto expresa una cierta regulación sobre las prácticas escolares. El curriculum prescripto u oficial sintetiza aquello que se debe enseñar, orienta en cómo se debe hacer, establece formas de vinculación entre los docentes, los estudiantes y el saber, etc. Aparece también una norma en el curriculum oculto o vivido que regula aquellas formas de comportamiento y valores que quedan por fuera del curriculum prescripto pero que se aprenden en la escuela.

En tercer lugar, al pensar al curriculum como norma, la autora problematiza los aspectos en común que pueden existir entre la vinculación de los sujetos con el curriculum como norma y aquélla otra que se da entre los sujetos y las normas sociales en las sociedades latinoamericanas. Si bien estas consideraciones representan una línea de indagación que se aleja de nuestros intereses actuales, resulta pertinente mencionarla por la conclusión que extrae la autora.

En este sentido, para Dussel, la historia de los países latinoamericanos, la influencia de las dictaduras, los regímenes democráticos que se han alternado, etc., conducen en líneas generales a una percepción de la norma como aquello que se enuncia para no cumplirse, para violarse o regular a los más débiles (4).

"[...] En términos de la normativa curricular, esto se expresa como el dejar a un lado, desconocer u "olvidar" lo que dice el currículum que debe enseñarse, en vez de discutir y confrontar para cambiar la norma, si es que creemos que es inadecuada o injusta [...]" (Dussel, 2010, p.6).

Esta línea de estudio aporta elementos para entender el funcionamiento real del curriculum en las instituciones educativas y sus efectos en la modificación o no de las prácticas educativas.

\section{La "programación de la enseñanza" como posible espacio de articulación entre la Didáctica y el Curriculum.}

Nos interesa en este apartado pensar y construir una posible vinculación entre la Didáctica y el Curriculum en torno al aporte que ambas pueden realizar 
Revista de la Escuela de Ciencias de la Educación, año 11, número 10, enero a diciembre de 2015. Páginas 149-166. ISSN 1851-6297. ISSN EN LINEA 2362-3349. DIDÁCTICA Y CURRICULUM: REFLEXIONES EN TORNO A LA CONSTITUCIÓN DE SU NORMATIVIDAD. SOFÍA PICCO

a la "programación de la enseñanza" y, por tanto, de las prácticas de enseñanza en su conjunto.

Barbosa Moreira (1999), Álvarez Méndez (2001) y Bolívar Botia (2008), entre otros autores, coinciden en que tradicionalmente el Curriculum se ha ocupado prioritariamente de qué enseñar, mientras que la Didáctica ha estado más inclinada a pensar el cómo enseñar.

Hay que mencionar también el origen geo-político de surgimiento de cada disciplina. El Curriculum prosperó en países anglosajones, con un sistema educativo básicamente descentralizado en su forma de gestión (5), mientras que la Didáctica se desarrolló más en Europa continental, con países que sustentaban una estructura centralizada en la organización de sus sistemas educativos.

En Argentina recibimos la influencia de la Didáctica proveniente de la tradición de Europa continental y sabemos que esta disciplina surge en estrecha vinculación con las preocupaciones acerca de cómo enseñar, dirigida al docente y cómo puede ayudarlo a mejorar sus prácticas. Pero sus preocupaciones siempre fueron más amplias que el método y abarcaron otras dimensiones de la enseñanza, como por ejemplo, las intenciones educativas -objetivos, fines, propósitos-, el contenido, la organización de la clase, los recursos, la evaluación de los aprendizajes y de la enseñanza, la formación docente, etc. (Cols, Amantea, Cappelletti y Feeney, 2002; Amantea, Cappelletti, Cols y Feeney, 2004; Camilloni, 2007).

Asimismo es cierto que la Didáctica ha mostrado mucho interés por el método de la enseñanza, llegando en algunos casos a desarrollarse un formalismo muy criticado y que muchas veces es el que se destaca al hablar de esta disciplina. Podríamos decir que la Didáctica que se desarrolla en las décadas del '60 y '70 es la más cuestionada por su instrumentalismo (Edelstein y Rodríguez, 1974; Becker Soares, 1985; Davini, 1996; Araujo, 2006).

En síntesis, esta compleja dinámica disciplinar nos lleva a coincidir con Camilloni (1996), cuando plantea que existen ciertas controversias acerca de si la Didáctica es una teoría de la enseñanza, como lo indica la tradición europea; si se confunde con la psicología educacional más propia de la tradición norteamericana; o si debe ser reemplazada por alguno de sus objetos de estudio, entre ellos el curriculum.

Por otra parte, los trabajos de Barbosa Moreira (1999) y de Álvarez Méndez (2001) abordan las complejas relaciones entre la Didáctica y la Curriculum sin realizar anclajes estrictos a las características que van adquiriendo a partir de su contextualización social y política. Esto nos permite sustentar el planteo anterior y, al mismo tiempo, pensar en una forma de vinculación que revierta en última instancia en un mejor aprovechamiento de categorías de análisis y herramientas de ambas disciplinas para los docentes y para las prácticas de enseñanza. 
Revista de la Escuela de Ciencias de la Educación, año 11, número 10, enero a diciembre de 2015. Páginas 149-166. ISSN 1851-6297. ISSN EN LINEA 2362-3349. DIDÁCTICA Y CURRICULUM: REFLEXIONES EN TORNO A LA CONSTITUCIÓN DE SU NORMATIVIDAD. SOFIA PICCO

Hay que reconocer que dependiendo de la definición inicial de Didáctica y de Curriculum que se adopte, se podrá hablar de que una disciplina es más amplia que la otra, que una abarca a la otra, o que son dos campos separados. No obstante, acordamos con autores como Barbosa Moreira (1999) y Bolívar Botia (2008) cuando dicen que la enseñanza se concreta en un marco curricular y que todo curriculum se efectiviza en las prácticas de enseñanza. Este planteo se encuentra con múltiples aspectos que se superponen entre la Didáctica y el Curriculum, cuestión que complejiza el análisis.

Es interesante la propuesta de vinculación de la Didáctica y el Curriculum expresada por Barbosa Moreira. "[...] Sugiero, entonces, que se construya, colectivamente, lo que Said denomina Programa de interferencia, en el cual se flexibilicen fronteras, se socialicen saberes restringidos a pequeños grupos de especialistas y se aborde el fenómeno educacional "secularmente" [...]" (1999, pp.29-30. El subrayado figura en cursiva en la edición consultada).

Esta propuesta permite poner entre paréntesis, podemos decir, las discusiones acerca de la delimitación de los campos y buscar una apertura al diálogo tendiente al mejoramiento de las prácticas de enseñanza a partir del intercambio de los sujetos involucrados en ella, sean éstos investigadores, teóricos, o maestros, prácticos.

Recuperando estos aportes, nos animamos a plantear que la "programación de la enseñanza" puede convertirse en un espacio de articulación entre la Didáctica y el Curriculum.

Entendemos la "programación de la enseñanza" como un complejo proceso que tiende a buscar la concreción en la práctica de las intenciones formativas formuladas en los diseños curriculares por las instancias centrales. En este sentido, la programación brinda una mayor contextualización o adecuación local, institucional y áulica a aquellas intenciones pedagógicas. Se corresponde, además, con la concepción y anticipación de la enseñanza e implica una serie de actividades de distinto tipo por parte del docente (Cols, 2004; Traverso, Picco y Coscarelli, 2011).

Cols identifica en la "programación de la enseñanza" así conceptualizada un requerimiento relativo a las características inherentes de la enseñanza en tanto práctica social. La enseñanza es una práctica intencional, racional y proyectual, por lo que la programación la constituye. "[...] Entonces, más que un procedimiento añadido para responder a las exigencias externas de control y comunicación, la programación obedece a un principio de estructuración y regulación interna [...]" (2004, p.6).

Asimismo, la autora diferencia otro "[...] requerimiento de orden organizativo y administrativo, ligado a la regulación de las prácticas de enseñanza y a la previsión de las formas de materializar y contextualizar una determinada propuesta de política curricular [...]" (2004, p.4). Hablar de la enseñanza institucionalizada en el contexto del sistema educativo lleva a pensar en la programación como 
Revista de la Escuela de Ciencias de la Educación, año 11, número 10, enero a diciembre de 2015. Páginas 149-166. ISSN 1851-6297. ISSN EN LINEA 2362-3349. DIDÁCTICA Y CURRICULUM: REFLEXIONES EN TORNO A LA CONSTITUCIÓN DE SU NORMATIVIDAD. SOFIA PICCO

una práctica que permite la supervisión, la previsión de lo que se va a hacer y la adquisición de un estado público de las acciones docentes vinculado a la responsabilidad institucional y social que se posee.

La actividad de enseñanza presenta tres rasgos que justifican la importancia de las tareas de programación. Primero, la enseñanza opera casi siempre en situaciones de restricciones (por ejemplo, de tiempo) y, por lo tanto, necesita asegurar algún grado de eficacia a sus acciones. En segundo término, la enseñanza es una actividad que se fija ciertos propósitos en relación con las situaciones que enfrenta y que utiliza o diseña técnicas adecuadas para conseguirlos. Por último, la enseñanza tiene que regular un ambiente bastante complejo y, por lo tanto, cuanto mayor cantidad de decisiones se tomen anticipadamente, mayor disponibilidad y capacidad de atención podrá dirigirse a la interacción directa (Feldman y Palamidessi, 2001, p.23).

Consideramos que la cita precedente pone en evidencia la importancia que posee la programación de la enseñanza. Las complejas prácticas de programación recuperan la centralidad del docente como sujeto responsable social e institucional de llevar a cabo la enseñanza. Además, el sentido amplio de programación que estamos aquí exponiendo, nos permite comprender en ella las instancias preactiva, interactiva y posactiva que ya identificara Jackson en 1968.

Por otra parte, Feldman y Palamidessi (2001) reconocen la diversidad de significados que se le asignan a los términos plan, planificación, programación, diseño, proyecto, etc. No obstante ello, sistematizan cuatro rasgos comunes a todos esos términos: 1- están asociados al propósito de resolver un problema que se presente en la práctica educativa; 2- se vinculan con la representación que cada uno de ellos encierra de un estado de cosas futuro que se desea alcanzar; 3- se relacionan con la posibilidad de anticiparse, de lanzarse hacia delante, desde el presente hacia el futuro, para lograr ciertos propósitos; y 4constituyen una hipótesis de trabajo, es decir, un intento, una prueba, que no sabemos si tendrá éxito hasta tanto no se eche a andar.

Para Feldman, la programación se vincula con aquellos aspectos más instrumentales de la enseñanza y, según sostiene, "[...] se puede decir que mediante la programación se pasa de la selección general del currículum al trabajo en clase [...]" (2010, p.42).

Podemos aludir aquí a aquellos "espacios de realización" (Salinas Fernández, 1997, p.35) que permiten analizar la enseñanza como una práctica social situada y condicionada. El docente al iniciar sus clases en un curso no se encuentra con un abanico de opciones ilimitadas, sino que tiene posibilidades y también restricciones para su ejercicio profesional. 
Revista de la Escuela de Ciencias de la Educación, año 11, número 10, enero a diciembre de 2015. Páginas 149-166. ISSN 1851-6297. ISSN EN LINEA 2362-3349. DIDÁCTICA Y CURRICULUM: REFLEXIONES EN TORNO A LA CONSTITUCIÓN DE SU NORMATIVIDAD. SOFIA PICCO

Sintéticamente, Salinas Fernández (1997) enumera cuatro aspectos básicos que condicionan la práctica docente: 1 - limitaciones de orden institucional; 2- el curriculum oficial; 3- el puesto de trabajo; y 4- los propios conocimientos, capacidades y epistemología del profesor.

Podríamos tomar los aportes de Cols (2004) para enriquecer los primeros dos aspectos. En este sentido, la autora recupera los planteos de Elmore y Sykes (1992) y de Eigenmann (1981) (6) para aludir a distintas características que pueden adoptar las políticas curriculares y, a su vez, analizar cómo las mismas pueden condicionar diferencialmente el accionar del docente con respecto a la programación.

Por un lado, una política curricular ambiciosa en términos de Elmore y Sykes o un diseño curricular de gran intensidad tal como lo expone Eigenmann, representa un documento con una importante cantidad de elementos prescriptos y se limitan por tanto las posibilidades de decisión de los docentes. Por otro lado, una alternativa modesta de política curricular o un diseño de menor intensidad, representa un curriculum que se halla a menor distancia del docente y su lugar de intervención en él es mayor. Esta alternativa prescribe acerca del contenido del curriculum y los estándares que deben ser alcanzados, pero pretende respetar los márgenes de autonomía del trabajo profesional del docente, al permitirle diferentes decisiones en torno a cómo enseñar aquellos contenidos que debe transmitir a sus alumnos.

En cualquiera de estos dos casos, encontramos que aparecen trabajos sobre curriculum o sobre política curricular, que estudian las prescripciones curriculares, cómo éstas son llevadas a la práctica y cómo interviene el docente en esa concreción crítica del curriculum. "Concreción crítica" en el sentido de concreción clave, central, en materia curricular; pero también en el sentido que Stenhouse (1991) le asignaba: el curriculum constituye aquellas intenciones educativas que se enuncian para ser trasladadas críticamente a la práctica, suponiendo que en ese traslado interviene el docente como pieza clave de la resignificación de lo prescripto para hacerlo realidad.

Es en este sentido que Cols analiza un proceso de programación complejo que se desarrolla en el ámbito institucional y que involucra una dimensión técnica pero básicamente una política y cultural. "[...] Dar forma y diseñar prácticas de enseñanza en contextos institucionales requiere procesos de deliberación, construcción de consensos y coordinación de acciones [...]" (2004, p.8).

Teniendo en cuenta lo antes expuesto y recordando la propuesta de articulación de la Didáctica y el Curriculum postulada por Barbosa Moreira (1999), consideramos que en la posibilidad de tomar y articular aportes de ambas disciplinas para el mejoramiento de la enseñanza es donde radica la riqueza de las relaciones interdisciplinares.

Antes de disputarse si la "programación de la enseñanza" es un objeto de estudio de una u otra disciplina, conceptualizarla como espacio de intersección 
Revista de la Escuela de Ciencias de la Educación, año 11, número 10, enero a diciembre de 2015. Páginas 149-166. ISSN 1851-6297. ISSN EN LINEA 2362-3349. DIDÁCTICA Y CURRICULUM: REFLEXIONES EN TORNO A LA CONSTITUCIÓN DE SU NORMATIVIDAD. SOFÍA PICCO

puede ayudar a intervenir en la enseñanza y colaborar con los docentes en la toma de decisiones informadas cotidianamente. Al mismo tiempo, esta propuesta nos conduce a reflexionar acerca de las características epistemológicas de la Didáctica y el Curriculum en la actualidad, específicamente en lo que respecta a su componente normativo.

\section{Características epistemológicas de la Didáctica y el Curriculum: pensar la normatividad en la actualidad.}

A partir de lo expuesto, consideramos que se torna fundamental reflexionar acerca de las características de la Didáctica y el Curriculum como disciplinas normativas en la actualidad. Como dejamos esbozado en otro trabajo (Picco, 2010a), aparece la necesidad de construir una perspectiva epistemológica tanto para la Didáctica como para el Curriculum que posibilite la articulación de aspectos normativo/prescriptivos y descriptivo/interpretativos. Estos aspectos pueden ser integrados en la reflexión sobre estas disciplinas, aún cuando han tenido una prevalencia alternada en la historia de las mismas, especialmente de la Didáctica.

En estudios realizados (Picco, 2010b; 2014), pudimos visualizar que diferentes perspectivas epistemológicas y metodológicas contemporáneas proponen la articulación de estos aspectos: por ejemplo, Schuster $(2001 ; 2007)$ para la filosofía de la ciencia y Marradi (2007) para la metodología. En líneas generales, estas propuestas persiguen la elaboración de normas, dirigidas a la orientación de las prácticas, conformadas -entre otros aspectos- a partir de considerar las características de la labor cotidiana que llevan a cabo los científicos.

La síntesis que se propone en este sentido para la generación de normas se encuentra bien representada cuando Frigerio expresa que una norma debe poseer una "textura abierta" (1991, p.27). Así, la norma didáctica podría representarse como una red en cuyos intersticios emergen la libertad y creatividad del docente, necesarias para su resignificación y para la apertura de aquellos espacios indeterminados de la práctica. Parafraseando a la autora, no hay normativa que pueda clausurar todos los significados que le pueden adjudicar los sujetos.

Específicamente en lo que respecta a la Didáctica, podemos mencionar algunos planteos que se generan en este momento de reconceptualización que está atravesando.

Salinas Fernández (1995), por ejemplo, sostiene que en la actualidad el discurso didáctico está atravesado por una serie de cuestiones significativas. Por un lado, nos encontramos con una expansión disciplinar importante en cuanto a la diversidad de producciones y temas concernientes al objeto de estudio, con el consecuente peligro de desdibujar el objeto así como también que el término pierda su capacidad de discriminación. 
Revista de la Escuela de Ciencias de la Educación, año 11, número 10, enero a diciembre de 2015. PÁginas 149-166. ISSN 1851-6297. ISSN EN LINEA 2362-3349. DIDÁCTICA Y CURRICULUM: REFLEXIONES EN TORNO A LA CONSTITUCIÓN DE SU NORMATIVIDAD. SOFIA PICCO

Por otro lado, el discurso de la Didáctica se encuentra en un estado de cierto "relativismo epistemológico" acompañado de un abandono del carácter propositivo de la teoría. La influencia de la corriente práctica y el lugar que en la misma se le asigna al docente como práctico reflexivo imprimió un giro a la disciplina, como vimos. Según expresa el autor, la Didáctica se encuentra en esta reubicación ante el desafío de cómo se puede enseñar -lo que representa el desarrollo de su faz normativa histórica-, pero queriendo diferenciarse de un discurso psicologicista -del que se deriva cómo enseñar a partir de saber cómo se aprende- y de otro socio-político crítico -que enfatiza la explicación de las funciones sociales de la escuela.

Según Salinas Fernández, la potencialidad explicativa, comprensiva o transformadora que en la actualidad los desarrollos teóricos de la Didáctica esgrimen como alternativa a la potencialidad normativa, lleva a la disciplina a una posición que puede caracterizarse por dos extremos igualmente novedosos. Por una parte la Didáctica se encuentra abandonando sus intenciones en cuanto al estudio y la regulación de las prácticas del aula, dejando de lado los esfuerzos comenianos en torno al método de enseñanza. Por otra parte, la Didáctica corre el riesgo de posicionarse en la construcción de un discurso crítico en torno a la educación como proceso socio-histórico de reproducción cultural.

Retomando la cuestión de la "base normativa" -parafraseando a Davini (1996; 2008)- es de interés situar el debate en los deslizamientos teóricos que Salinas Fernández conceptualiza para la Didáctica en los últimos años.

"[...] resulta complicado elaborar un discurso didáctico que, recién abandonado el reduccionismo psicologicista (cómo aprende el niño según las últimas investigaciones y, por tanto, cómo se debe enseñar), se sitúa ante una teoría sociológica crítica (qué funciones desempeña la escuela en las sociedades capitalistas postindustriales) y a partir de ello, o a pesar de ello, se enfrenta ante el problema de cómo se puede enseñar" (1995, p.54).

Candau (1996), por su parte, propone la revisión crítica de una didáctica instrumental y la construcción de una didáctica fundamental. La primera está conformada por un conjunto de reglas de carácter universal acerca del cómo hacer, desvinculado del contexto social y político en el que se lleva a cabo la enseñanza, de los contenidos que se transmiten y de los fines que se persigue. En la crítica a esta didáctica tecnicista resta el desafío de la construcción de un campo disciplinar -que la autora denomina didáctica fundamental-sustentado en al menos cuatro pilares: 1- la consideración del proceso de enseñanza y de aprendizaje desde una perspectiva multidimensional que contemple la faz técnica, humana y política; 2 - la explicitación de los supuestos teóricos, epistemológicos, metodológicos que subyacen a la construcción del conocimiento; 3- la necesaria relación teoría y práctica; y 4- la búsqueda de una eficiencia 
Revista de la Escuela de Ciencias de la Educación, año 11, número 10, enero a diciembre de 2015. Páginas 149-166. ISSN 1851-6297. ISSN EN LINEA 2362-3349. DIDÁCTICA Y CURRICULUM: REFLEXIONES EN TORNO A LA CONSTITUCIÓN DE SU NORMATIVIDAD. SOFÍA PICCO

pedagógica no desgajada de una transformación social adecuada a la mayoría de la población del país.

Sin profundizar en otro tipo de comparación, nos presentamos una similitud entre Salinas Fernández (1995) y Candau (1996) en el punto de postular algunas características comunes para el campo de la Didáctica. Ambos autores están en contra de una disciplina que postule reglas universales acerca del cómo hacer en las prácticas de enseñanza. Pero el abandono de este tipo de pretensiones no está acompañado por una renuncia del componente normativo. Por el contrario, postulan una disciplina que intervenga, que posea un discurso de carácter propositivo, que diga cómo actuar en las prácticas, porque es adecuado en un determinado contexto áulico, institucional y social.

Por otro lado, Davini $(1996,2008)$ plantea que las necesidades de las prácticas de enseñanza y de los docentes demandan a la Didáctica establecer ciertos criterios básicos y metodológicos para la acción, pero esta disciplina, en los últimos tiempos, se ha distanciado de estas intencionalidades. En este corrimiento de la función normativa de la Didáctica, además de los factores y procesos internos de la dinámica disciplinar, la autora enuncia algunos otros que denomina extra didácticos y que contribuyen a esta situación, a saber: el fracaso de las utopías modernas, la crisis del sistema educativo y el debilitamiento del discurso y las propuestas de la Pedagogía.

No obstante, es necesario que la Didáctica brinde dichos criterios para la orientación de la acción y de los sujetos, permitiéndoles a estos últimos hacer ejercicio de su profesionalidad, tendiendo al mejoramiento de la enseñanza. La ausencia de estas intervenciones didácticas convalidaría una práctica educativa artesanal o intuitiva, en abierta contradicción con un punto esencial que se refiere a la educación como proceso social y de carácter público, por lo tanto,

En la nueva perspectiva de la didáctica debiera plantearse, respecto de la normativa, un mínimo de acuerdo que, aun reconociendo que supone marcos teóricos a probar, represente conocimientos públicos, es decir que puedan compartir y utilizar otras personas y no sean patrimonio de la intuición intransferible del sujeto que investiga o que enseña (Davini, 1996, p.51).

También es interesante la forma en la que Cols (2003) plantea este desafío para la Didáctica. Nuestra disciplina debe trabajar en la construcción de principios con cierto grado de generalidad, referidos a la buena enseñanza en articulación con interpretaciones sobre el trabajo del docente en el aula y la manera en la que se lleva a cabo la enseñanza.

Por su parte, Araujo (2006) sostiene que la producción Didáctica de la década del '90 contempla el dilema explicación-proyección/proposición aunque deja de lado el tratamiento universal del método y la prescripción uniforme para el desarrollo eficaz de la enseñanza. 
Revista de la Escuela de Ciencias de la Educación, año 11, número 10, enero a diciembre de 2015. Páginas 149-166. ISSN 1851-6297. ISSN EN LINEA 2362-3349. DIDÁCTICA Y CURRICULUM: REFLEXIONES EN TORNO A LA CONSTITUCIÓN DE SU NORMATIVIDAD. SOFIA PICCO

En lo que respecta al Curriculum, Gimeno Sacristán (2012) realiza un breve repaso histórico y concluye que en la actualidad se destaca como problemática el carácter regulador del curriculum con respecto a las prácticas educativas. Este proyecto de formación implica, como dice el autor, una confianza en la educación, en sus posibilidades de transformación de esos sujetos, en la concreción de las intenciones y deseos formativos que sintetiza. El curriculum también conlleva una regulación sobre las prácticas de enseñanza, sobre cómo podrían o deberían llevarse a cabo y sobre qué se debería enseñar. El curriculum, aún cuando lo veamos como algo naturalizado en nuestra vida cotidiana, es un invento social que se ha ido asociando fuertemente a la educación escolar y que la organiza en términos de sujetos, espacios, tiempos y saberes que circulan -y que no circulan- en ella.

Asimismo, tanto para el caso del curriculum como para los niveles más específicos de programación de la enseñanza, es importante reflexionar sobre los aspectos normativos en una racionalidad crítica. Esta tensión es difícil de resolver, sobre todo si tomamos los aportes de Bolívar Botia (1999) cuando expresa que las teorías críticas pueden ser mejor pensadas como una plataforma con categorías para el análisis minucioso que como propuestas sistemáticas de intervención, vertiente esta última que iría en contra de los sentidos de la crítica.

\section{Consideraciones finales.}

Postular que la "programación de la enseñanza" puede ser ese espacio de intersección entre la Didáctica y el Curriculum, conlleva dos desafíos nodales sobre los que, según entendemos, es prioritario trabajar hoy.

En primer lugar, abre un diálogo interdisciplinario en vistas al mejoramiento de las prácticas de enseñanza. Tal como expusimos, la "programación de la enseñanza" es una práctica central del accionar profesional del docente y, por tanto, se convierte en un espacio potencial para que la Didáctica y el Curriculum realicen aportes conceptuales e instrumentales significativos.

En segundo lugar y como consecuencia de lo anterior, podemos decir que articular la Didáctica y el Curriculum en torno a la "programación de la enseñanza", nos lleva a reflexionar acerca de la constitución del componente normativo en estas disciplinas como normativas. La integración equilibrada entre explicación, normas, valores y prácticas debe ser parte de la perspectiva epistemológica que se construya en la actualidad.

Asimismo, a partir de lo expuesto podemos plantear que la Didáctica, el curriculum y la escuela se unen en la representación y en la concreción de la utopía educativa de la modernidad. Pero debemos seguir pensando en nuevas opciones de racionalidad que nos permitan construir disciplinas normativas como la Didáctica y el Curriculum, preocupadas por el mejoramiento de las prácticas de enseñanza, en un contexto posmoderno. 
Revista de la Escuela de Ciencias de la Educación, año 11, número 10, enero a diciembre de 2015. Páginas 149-166. ISSN 1851-6297. ISSN EN LINEA 2362-3349. DIDÁCTICA Y CURRICULUM: REFLEXIONES EN TORNO A LA CONSTITUCIÓN DE SU NORMATIVIDAD. SOFÍA PICCO

\section{Notas bibliográficas:}

(1) Comenio había comenzado a escribir la Didáctica Magna en lengua checa en 1627 (Ravecq, 1957). La edición sueca se publicó en 1632 y la edición latina en 1657. Posiblemente sea esta última la más divulgada. Recordamos que el latín era la lengua de los intelectuales hasta el siglo XVIII y la traducción a ella contribuía con la difusión de las obras.

(2) Cabe aclarar que la expresión "sujetos normados" nos pertenece ya que en la edición consultada del libro de von Wright se los denomina "sujetos normativos". Según entendemos, los "sujetos normativos" son más bien aquéllos que emiten o fijan las prescripciones y no quienes son regidos por ellas.

(3) Como un ejemplo actual de esta indiscriminación podemos hacer referencia al Marco General de Política Curricular vigente para la Provincia de Buenos Aires que abarca a todos los niveles y modalidades del sistema educativo, en el que se explicita y fundamenta que "[...] los nuevos diseños curriculares elaborados a partir de la Ley de Educación Provincial $N^{\circ} 13.688$ [...] se asumen como comunes, prescriptivos, paradigmáticos y relacionales [...]" (2007, p.15. La negrita figura en la edición consultada). Analizando la caracterización que se brinda para "prescriptivos" encontramos rasgos que en los términos que estamos utilizando, la vincularían a lo normativo, como por ejemplo cuando se hace referencia al lugar del docente en la interpretación y uso profesional de los Diseños Curriculares para la programación de sus clases. (Para más información ver el informe final del proyecto "Programación de la enseñanza en la escuela primaria: discursos y prácticas", Programa de Incentivos a la Investigación, Ministerio de Ciencia, Tecnología e Innovación Productiva de la Nación, H588).

(4) Dussel recupera el trabajo de: O’Donnell, G. (2002). "Las poliarquías y la (in)efectividad de la ley en América Latina”. Méndez, G. O’Donnell y P. S. Pinheiro (eds.), La (in)efectividad de la ley y la exclusión en América Latina. Buenos Aires: Paidós.

En una línea similar aunque no estrictamente pertinente a los fines de este trabajo, se puede consultar el trabajo de Fumagalli (2008).

(5) Hamilton (1999) plantea como una paradoja que la Didáctica no tenga un desarrollo relevante, como es el caso del Curriculum, en los países anglosajones. Según explica, los primeros desarrollos de Didáctica acentuaron el carácter formalista de la disciplina y la fueron volcando hacia ser considerada como un conjunto de procedimientos para la transmisión eficaz del curriculum, quedando como una disciplina secundaria.

(6) La autora refiere a las siguientes obras: Elmore, R. y Sykes, G. (1992). "Curriculum Policy". En: Jackson, Ph. (ed.). Handbook of Research on Curriculum. New York, Macmillan. Y Eigenmann, J. (1981): El desarrollo secuencial del curriculum. Madrid, Anaya.

\section{Referencias bibliográficas}

- $\quad$ Álvarez Méndez, J. M. (2001). Entender la Didáctica, entender el Curriculum. Buenos Aires: Miño y Dávila.

- Amantea, A., Cappelletti, G., Cols, E. \& Feeney, S. (2004). "Concepciones sobre curriculum, el contenido escolar y el profesor en los procesos de elaboración de textos curriculares en Argentina". En Archivos Analíticos de Política Educativa, 12(40).

- $\quad$ Araujo, S. (2006). Docencia y enseñanza. Una introducción a la didáctica. (Cuadernos Universitarios) No. 13. Bernal, Buenos Aires: Universidad Nacional de Quilmes.

- Barbosa Moreira, A. F. (1999). "Didáctica y curriculum: cuestionando fronteras". En Propuesta Educativa. Novedades Educativas, FLACSO, Año 10, (20), 24-30. 
Revista de la Escuela de Ciencias de la Educación, año 11, número 10, enero a diciembre de 2015. Páginas 149-166. ISSN 1851-6297. ISSN EN LINEA 2362-3349. DIDÁCTICA Y CURRICULUM: REFLEXIONES EN TORNO A LA CONSTITUCIÓN DE SU NORMATIVIDAD. SOFIA PICCO

- $\quad$ Barco de Surghi, S. (1989). Estado actual de la pedagogía y la didáctica. En Revista Argentina de Educación. Asociación de Graduados en Ciencias de la Educación, Año VII, N ${ }^{\circ}(12), 7-23$.

- Basabe, L. \& Cols, E. (2007). "La enseñanza”. En Camilloni, A. (Comp.) (2007). El saber didáctico. Buenos Aires: Paidós.

- $\quad$ Becker Soares, M. (1985). "Didáctica, una disciplina en busca de su identidad". En Revista A.N.D.E. Universidad Federal de Mina Gerais, Año 5, (9), 1-9.

- Bolívar Botia, A. (1999). "Modelos de diseño curricular de corte crítico y postmoderno: descripción y balance". En Escudero Muñoz, J. M. (Ed.), Diseño, desarrollo e innovación del curriculum. Madrid: Síntesis.

- Bolívar Botia, A. (2008). Didáctica y curriculum: de la modernidad a la posmodernidad. Málaga: Aljibe.

- Camilloni, A. (1993). "Epistemología de la didáctica de las ciencias sociales". En Aisenberg, B. \& Alderoqui (Comps.). Didáctica de las ciencias sociales. Aportes y reflexiones. Buenos Aires: Paidós.

- $\quad$ Camilloni, A., Davini, M. C., Edelstein, G., Litwin, E., Souto, M. \& Barco, S. (1996). Corrientes didácticas contemporáneas. Buenos Aires: Paidós.

- $\quad$ Camilloni, A. (Comp.) (2007), El saber didáctico. Buenos Aires: Paidós.

- Candau, V. M. (1996). "A revisăo da Didática". En Candau, Vera Maria (Org.), Rumo a uma nova didática. Petrópolis: Vozes.

- $\quad$ Cols, E. (2003). El campo de la didáctica: recorrido histórico y perspectivas actuales. Ficha de circulación interna de la cátedra "Didáctica", Facultad de Humanidades y Ciencias de la Educación, Universidad Nacional de La Plata.

- Cols, E. (2004). Programación de la enseñanza. Ficha de la cátedra de "Didáctica I", Facultad de Filosofía y Letras, Universidad de Buenos Aires Oficina de Publicaciones de la Facultad de Filosofía y Letras.

- Cols, E., Amantea, A., Cappelletti, G. \& Feeney, S. (2002). Los procesos de diseño curricular en la Argentina: diversidad de tradiciones sobre el curriculum, el contenido y el profesor. Trabajo realizado en el marco del Proyecto UBACyT F015 "Tendencias en el diseño y desarrollo del currículum" (Período 2000-2002), directora Alicia Camilloni. Universidad de Buenos Aires, Facultad de Filosofía y Letras, IICE. Mimeo.

- Davini, M. C. (2008). Métodos de enseñanza. Didáctica general para maestros y profesores. Buenos Aires: Santillana.

- De Alba, A. (1995). Curriculum: Crisis, Mito y Perspectivas. Buenos Aires: Miño y Dávila.

- Díaz Barriga, Á. (1991). Didáctica y curriculum. Convergencias en los programas de estudio. México: Ediciones Nuevomar S.A. de C.V.

- Díaz Barriga, Á. (1994). El currículo escolar. Surgimiento y perspectivas. Buenos Aires: Rei Argentina; Instituto de Estudio y Acción Social; Aique.

- Díaz, E. (1997). "Conocimiento, ciencia y epistemología”. En Díaz, E. (Ed.): Metodología de las ciencias sociales. Buenos Aires: Biblos.

- Dussel, I. (2010). El curriculum. Explora. Las ciencias en el mundo contemporáneo. Programa de Capacitación Multimedial, Ministerio de Educación de la Nación. Recuperado de http://explora.educ.ar/wp-content/uploads/2010/04/PEDAG07-EIcurriculum.pdf, 03/05/2012.

- $\quad$ Edelstein, G. \& Rodríguez Ousset, A. (1974). El método: factor definitorio y unificador de la instrumentación didáctica. En Revista de Ciencias de la Educación, Año IV, (12), 21-33.

- $\quad$ Egan, K. (2000): Mentes educadas. Barcelona: Paidós. 
Revista de la Escuela de Ciencias de la Educación, año 11, número 10, enero a diciembre de 2015. Páginas 149-166. ISSN 1851-6297. ISSN EN LINEA 2362-3349. DIDÁCTICA Y CURRICULUM: REFLEXIONES EN TORNO A LA CONSTITUCIÓN DE SU NORMATIVIDAD. SOFÍA PICCO

- $\quad$ Feldman, D. \& Palamidessi, M. (2001). Programación de la enseñanza en la universidad. Problemas y enfoques. Colección Universidad y Educación: Serie Formación Docente № 1 Universidad Nacional de General Sarmiento.

- Feldman, D. (2010). Didáctica general. Aportes para el desarrollo curricular: Instituto Nacional de Formación Docente (INFod). Argentina: Ministerio de Educación. Recuperado de http://www.me.gov.ar/infod/documentos/didactica_general.pdf, 26/06/2010.

- $\quad$ Fenstermacher, G. D. (1989). "Tres aspectos de la filosofía de la investigación sobre la enseñanza”. En Wittrock, M. C. La investigación de la enseñanza, I. Enfoques, teorías y métodos. Madrid: Centro de Publicaciones Ministerio de Educación y Cultura y Paidós Ibérica.

- $\quad$ Frigerio, G. (1991). "Curriculum: norma, intersticios, transposición y textos". En Frigerio, G. (Comp.), Curriculum presente ciencia ausente. Normas, teorías y críticas. Buenos Aires: Miño y Dávila; FLACSO.

- Gimeno Sacristán, J. (2012). “¿Qué significa el curriculum?”. En Gimeno Sacristán, J., Feito Alonso, R., Perremoud, P. \& Clemente Linuesa, M. Diseño, desarrollo e innovación del curriculum. Madrid: Morata.

- Hamilton, D. (1993). Orígenes de los términos educativos 'clase' y 'curriculum'. En Revista Iberoamericana de Educación "Estado y Educación", (1). Recuperado de www. campus-oei/revista/index.html

- Hamilton, D. (1999). La paradoja pedagógica (O: ¿por qué no hay una didáctica en Inglaterra?). En Revista Propuesta Educativa, (Año 10, (20), 6-13.

- Jackson, P. (1992). La vida en las aulas. Madrid: Morata.

- Kemmis, S. (1993). El curriculum: más allá de la teoría de la reproducción. Madrid: Morata.

- Klimovsky, G. \& Hidalgo, C. (1998). La inexplicable sociedad. Cuestiones de epistemología de las ciencias sociales. Buenos Aires: A-Z Editora.

- $\quad$ Lundgren, U. P. (1992). Teoría del Curriculum y Escolarización. Madrid: Morata.

- Marradi, A. (2007). "Método, metodología, técnicas". En Marradi, A., Archenti, N. \& Piovani, J. I. Metodología de las ciencias sociales. Buenos Aires: Emecé.

- Martínez Bonafé, J. (2004). Esfera pública, conocimiento escolar y didáctica crítica. $X$ Congreso Fedicaria, Valencia. Recuperado de http://www.fmrppv.org/eets/fedi.doc, 15/06/2007.

- Picco, S. (2010a). Reflexiones en torno a la normatividad en la didáctica general. En Revista Teoría y Didáctica de las Ciencias Sociales, Escuela de Educación, Facultad de Humanidades y Educación, Universidad de los Andes, Mérida, Venezuela. Número 16 de Enero-Julio de 2010, pp.207-227. Con referato. I.S.S.N. No 1316-9505. Recuperado de http://www.saber.ula.ve/handle//123456789/33628

- Picco, S. (2010b). La normatividad en discusión: El caso de la didáctica a partir de debates epistemológicos y metodológicos contemporáneos. (En línea). Tesis de la Maestría en Ciencias Sociales, publicada en el repositorio institucional "Memoria Académica", Facultad de Humanidades y Ciencias de la Educación, Universidad Nacional de La Plata. Recuperado de http://www.memoria.fahce.unlp.edu.ar/tesis/te.390/te.390.pdf

- $\quad$ Picco, S. (2014). Concepciones en torno a la normatividad en la didáctica. Un análisis interdisciplinario de obras teóricas didácticas y curriculares en la Argentina entre 1960 y 1990. (En línea). Tesis del Doctorado en Ciencias de la Educación, Facultad de Humanidades y Ciencias de la Educación, Universidad Nacional de La Plata, publicada en el repositorio institucional de la UNLP "SEDICl". Disponible en: http://sedici.unlp.edu. ar/handle/10915/35590 
Revista de la Escuela de Ciencias de la Educación, año 11, número 10, enero a diciembre de 2015. Páginas 149-166. ISSN 1851-6297. ISSN EN LINEA 2362-3349. DIDÁCTICA Y CURRICULUM: REFLEXIONES EN TORNO A LA CONSTITUCIÓN DE SU NORMATIVIDAD. SOFIA PICCO

- $\quad$ Salinas Fernández, B. (1995). "Límites del discurso didáctico actual". En AA.VV. Volver a pensar la educación. Prácticas y discursos educativos. (Congreso Internacional de Didáctica). España: Fundación Paideia; Morata.

- Salinas Fernández, D. (1997). "Curriculum, racionalidad y discurso didáctico". En Poggi, M. (Comp.), Apuntes y aportes para la gestión curricular. Buenos Aires: Kapelusz.

- Schuster, F. L. (2001). Exposición. En Schuster, Federico L., Giarraca, N., Aparicio, S., et al. El oficio de investigador. Buenos Aires: Homo Sapiens; Instituto de Investigaciones en Ciencias de la Educación, Facultad de Filosofía y Letras, Universidad de Buenos Aires.

- Schuster, F. L. (2007). Presentación del libro: MARRADI, Alberto, ARCHENTI, Nélida, y PIOVANI, Juan Ignacio. (2007). Metodología de las ciencias sociales. Buenos Aires: Emecé.

- $\quad$ Stenhouse, L. (1991). Investigación y desarrollo del curriculum. Madrid: Morata.

- Traverso, O. V.; Picco, S. \& Coscarelli, M. R. (2011). La programación de la enseñanza y las mediaciones de la práctica docente. Ponencia presentada en el Congreso Comunicación/Ciencias Sociales desde América Latina: tensiones y disputas en la producción de conocimiento para la transformación, (Comcis), Facultad de Periodismo y Comunicación Social, Universidad Nacional de La Plata. 30-08 al 02-09-2011. Publicación de la ponencia en $C D$.

- Von Wright, G. H. (1970). Norma y acción. Una investigación lógica. Madrid: Tecnos. 
